\title{
Metaanálisis de la validez del inmunodiagnóstico del virus linfotrópico de células T humanas ı/॥ en bancos de sangre, 2000-2018
}

\author{
Meta-analysis regarding the validity of the immunodiagnosis of human T-cell \\ lymphotropic virus I/II in blood banks, 2000-2018
}

\section{Meta-análise da validade do imunodiagnóstico do vírus linfotrópico de células t humanas i/ii em bancos de sangue, 2000-2018}

\author{
Claudia Patricia Orrego-Marín'; Jaiberth Antonio Cardona-Arias² \\ 1 MSc Microbiología. Universidad de Antioquia, Medellín, Colombia. claudiorrego29@hotmail.com. orcid: https://orcid.org/0000-0001- \\ 8672-5552 \\ 2 MSc Epidemiología, MSc Economía aplicada. Universidad de Antioquia. jaiberth.cardona@udea.edu.co. oRcid: https://orcid.org/0000- \\ 0002-7101-929X
}

Recibido: 28/09/2020. Aprobado: 3/06/2021. Publicado: 14/07/2021

Orrego-Marín CP, Cardona-Arias JA. Metaanálisis de la validez del inmunodiagnóstico del virus linfotrópico de células T humanas i/ii en bancos de sangre, 2000-2018. Rev. Fac. Nac. Salud Pública. 2021;39(3):e343861. Dor: https://doi. org/10.17533/udea.rfnsp.e343861

\section{Resumen}

Objetivo: Evaluar la validez del inmunodiagnóstico del HTLV I/II en bancos de sangre, con base en estudios publicados en la literatura científica. Metodología: Se efectuó un metaanálisis de pruebas diagnósticas siguiendo la guía PRISMA y las recomendaciones de Cochrane. Se evaluó la calidad metodológica con QUADAS y se garantizó la reproducibilidad y la exhaustividad. Se realizó también un análisis de efectos aleatorios para la sensibilidad, la especificidad, los cocientes de probabilidad, la razón de momios diagnóstica y la curva característica operativa del receptor (ROC) con sus intervalos de confianza (IC) del 95 \%. Resultados: Se tamizaron 4604 estudios, de los cuales solo tres cumplieron el protocolo. Se evaluaron 548 infectados con HTLV I/II y 6643 sanos. El inmunodiagnóstico de HTLV I/II presentó una sensibilidad del 99
$\%$ (IC95 \% = 98,0-99,0), especificidad del $100 \%$ (IC95 \% = 99,9-100), cocientes de probabilidad positivo de 315,8 (IC95 \% $=128,2-778,5)$ y negativo de $0,02($ IC95 \% = 0,01-0,04), razón de momios diagnóstica de 24373 (IC95 \% = 6864-86545) y área bajo la curva Roc del 99,9\%. Conclusión: Se dispone de pocos estudios en este campo del inmunodiagnóstico HTLV I/II. El elevado número de sujetos analizados evidenció alta validez del inmunodiagnóstico, lo que resulta determinante para garantizar la inocuidad de las unidades de sangre, la detección de portadores asintomáticos, la disminución de la transmisión y el inicio de tratamiento.

-Palabras clave: Banco de sangre, metaanálisis, prevención y control, pruebas inmunológicas, virus linfotrópico de células T humanas tipo I y tipo II (HTLV I/II). 


\section{Abstract}

Objective: To evaluate the validity of the immunodiagnosis of HTLV I/II in blood banks, based on studies published in the scientific literature. Methodology: A metaanalysis of diagnostic tests was carried out following the PRISMA guidelines and Cochrane recommendations. The methodological quality was evaluated with QUADAS, and reproducibility and completeness were guaranteed. A random effects analysis was also performed with respect to sensitivity, specificity, likelihood ratios, diagnostic odds ratio, and receiver operating characteristic curve (ROC) with their $95 \%$ confidence intervals (CI). Results: 4,604 studies were screened, of which only three complied with the protocol. 548 subjects infected with HTLV I/II and 6,643 healthy subjects were evaluated. The immunodiagnosis of HTLV I/II had a sensitivity of $99 \%$ ( $95 \% \mathrm{CI}=98.0-99.0)$, a specificity of 100 $\%(95 \% \mathrm{CI}=99.9-100)$, a positive likelihood ratio of 315.8 $(95 \% \mathrm{CI}=128.2-778.5)$ and a negative likelihood ratio of 0.02 ( $95 \% \mathrm{CI}=0.01-0.04)$, a diagnostic odds ratio of 24,373 (95\% $\mathrm{CI}=6,864-86,545)$, and an area under the ROC curve of 99.9 $\%$. Conclusion: Few studies are available in the field of HTLV I/II immunodiagnosis. The high number of subjects analyzed showed high validity of the immunodiagnosis, which is decisive to guarantee the safety of the blood units, the detection of asymptomatic carriers, the decrease in transmission, and the start of treatment.

--Keywords: blood bank, meta-analysis, prevention and control, immunological tests, human T-cell lymphotropic virus type I and type II (HTLV I/II).

\section{Resumo}

Objetivo: Avaliar a validade do imunodiagnóstico do HTLV I/II nos bancos de sangue, baseados nos estudos publicados na literatura científica. Metodologia: Foi realizada uma meta-análise de testes diagnósticos seguindo a guia PRISMA e as recomendações de Cochrane. Foi avaliada a qualidade metodológica com QUADAS e garantiu-se a reprodutibilidade e a integridade. Realizou-se também uma análise de efeitos aleatórios para a sensibilidade, a especificidade, os quocientes de probabilidade, a razão de probabilidade diagnóstica e a Curva Característica de Operação do Receptor (Curva ROC) com seus Intervalos de Confiança (IC) de 95\%. Resultados: Foram selecionados 4604 estudos, dos quais somente 3 cumpriram com o protocolo. Foram avaliados 548 infectados com o vírus HTLV I/II e 6.643 saudáveis. O imunodiagnóstico de HTLV I/II apresentou uma sensibilidade de $99 \%$ (IC95\% = 98,0-99,0), especificidade de 100\% (IC95\%= 99,9-100), quocientes de probalidade positiva de 315,8 (IC95\% $=128,2-778,5)$ e negativo de $0,02($ IC95\% $=0,01-0,04)$, razão de probabilidade diagnóstica de $24373($ IC95\% $=6864-86545)$ e área sob a curva ROC de 99,9\%. Conclusão: São poucos os estudos disponíveis neste campo do imunodiagnóstico HTLV I/II. $\mathrm{O}$ elevado número de pessoas analisadas evidenciou alta validade do imunodiagnóstico, o que é decisivo para garantir a inocuidade das unidades de sangue, a detecção de portadores assintomáticos, a diminuição da transmissão e 0 início do tratamento.

--------Palavras-chave: Banco de sangue; Meta-análise; Prevenção e controle; Testes imunológicos; Vírus linfotrópico de células T humanas tipo I e tipo II (HTLV I/II).

\section{Introducción}

Los virus linfotrópicos de células $\mathrm{T}$ humanas (human T-cell lymphotropic virus, HTLV) tipo I y tipo II son los primeros retrovirus identificados en humanos en la década de los ochenta del siglo xx. Estos virus comparten aproximadamente el $60 \%$ de homología genética, están clasificados como oncovirus por su patogenicidad, sus células blanco son los linfocitos T CD4+ y CD8+ [15], y su transmisión ocurre por vía sexual, vertical, parenteral o transfusiones sanguíneas [2-4,6,7]. El HTLV-I causa enfermedades como leucemia, linfoma de células $\mathrm{T}$ adultas, mielopatía asociada al HTLV o paraparesia espástica tropical; además, se asocia con artropatía inflamatoria crónica, carcinomas, cistitis crónica, dermatitis infecciosa, enfermedad linfoproliferativa, polimiositis, síndrome de Sjögren, síndrome inflamatorio, tiroiditis, uveítis, aumento en la mortalidad general por cáncer y riesgo de infecciones bacterianas, principalmente en tórax, vejiga y riñón $[3,4,6-8]$.

Actualmente, se estiman entre 10 y 20 millones de personas infectadas en todo el mundo, incluyendo habitantes de países no endémicos, como resultados de los procesos migratorios $[4,5,7,8]$. Existe una alta prevalencia de la infección en Japón, Melanesia, Australia, países africanos, Irán, América del Norte, islas del Caribe, Brasil, Chile, Colombia, Paraguay Perú, Guayana Francesa, Surinam y Venezuela [2,3,7-11]. La mayoría de casos de infección permanece asintomática; solo del 1 al $5 \%$ desarrollan leucemia de células $\mathrm{T}$ adultas y entre el $3 \mathrm{y}$ 
el $5 \%$ mielopatía asociada al HTLV-I $[4,8]$, lo que depende del periodo de incubación, que en algunos casos es desconocido y en otros puede tardar décadas [4]. Este largo periodo de incubación es relevante para la medicina transfusional, dado el alto riesgo de transmisión por esta vía, donde la seroconversión puede ser entre el 40 y el $60 \%$ de los receptores de productos sanguíneos [5].

En ausencia de una vacuna preventiva y con opciones limitadas de tratamiento, las medidas preventivas se centran en una detección oportuna y en la prevención de su transmisión. Pese a esto, la infección por HTLV I/II sigue siendo difícil de diagnosticar, principalmente por características como el curso asintomático de la mayoría de casos y su asociación con otras alteraciones neurológicas [6,7]. Debido a esto, en países como Japón se incorporó, desde 1986, la detección del HTLV I/II en todos los donantes de sangre, política que posteriormente se adoptó en Estados Unidos en 1988, en Canadá en 1989, y en Francia y algunos países europeos y sudamericanos a partir de 1991. Con este tipo de medidas se ha logrado el aumento de casos identificados y posterior disminución de la prevalencia del HTLV I/II, que en países desarrollados oscila entre 1/10 000 y 1/100 000 habitantes, con un riesgo de transmisión de alrededor de 2 a 63 por millón de habitantes. Luego de la incorporación de las pruebas de tamización serológica en los bancos de sangre ha disminuido la transmisión por esta vía en más del $95 \%[3,5,6]$.

En los bancos de sangre, la detección de HTLV I/ II se realiza mediante la identificación de anticuerpos con inmunoensayo ligado a enzima (enzyme-linked immunosorbent assay, ELISA), con posterior confirmación por Western blot. Según la Organización Mundial de la Salud (OMS), una muestra es positiva cuando presenta alguna banda específica de los genes env, como gp46 o gp62/68, o de los genes gag, como p19, p24 o p53; sin embargo, existen otros criterios, como los de la Red Europea de Investigación, que exigen la presencia de las bandas correspondientes a p19 y p24 del gen gag, y las proteínas de la envoltura viral rgp21 y rgp46-I [11].

A pesar de los diferentes criterios establecidos por la oms y la Red Europea de Investigación, la tamización de los virus HTLV I/II ha mostrado gran dificultad, dado los resultados discordantes entre pruebas. Por ejemplo, Morimoto et al., en 2007, compararon dos pruebas de detección serológica del HTLV I/II (inmunoensayo enzimático) con antígenos diferentes: una con lisados virales purificados de HTLV I/II asociados con el antígeno recombinante p21E de HTLV-I (Vironostika TM HTLV I/II), y una prueba recombinante con péptidos sintéticos de las proteínas de envoltura de HTLV I/II asociados con una proteína recombinante transmembrana de HTLV-II (Murex TM HTLV I/II). Al compararse con Western blot, ambos ensayos presentaron sensibilidad del $100 \%$; pero al com- pararse con una Polymerase Chain Reaction (PCR), la sensibilidad fue del 100 y el $89 \%$, respectivamente [12].

El curso asintomático del HTLV I/II y el difícil acceso al diagnóstico evidencian la relevancia de los bancos de sangre para la detección y la vigilancia epidemiológica de esta infección. Es por ello fundamental contar con tecnología con alta validez en las pruebas, que permita identificar el verdadero estado de salud de los individuos y evitar el diferimiento innecesario de donantes potenciales, dado que esto representa un riesgo para garantizar un suministro suficiente de sangre y elevados costos económicos para el banco de sangre $[6,8]$.

Por lo anterior, el objetivo de esta investigación fue evaluar la validez del inmunodiagnóstico del HTLV I/II en bancos de sangre, con base en estudios publicados en la literatura científica mundial, dado que este tipo de revisiones mejora la validez externa de los resultados, genera recomendaciones clínicas y epidemiológicas de mayor grado de evidencia, disminuye sesgos y errores aleatorios, permite conocer el estado actual de la investigación en un campo y consolidar hipótesis para investigaciones posteriores [13].

\section{Metodología}

Se realizó un estudio teórico o basado en estudios secundarios, aplicando las fases y los criterios de rigor metodológico de las revisiones sistemáticas de la literatura científica, caracterizadas por su sistematicidad (la búsqueda y selección de estudios se fundamenta en una pregunta de investigación definida a priori), reproducibilidad (de la búsqueda y selección de los estudios, así como de la extracción de la información de los artículos seleccionados), exhaustividad (el protocolo de la revisión busca captar todas las publicaciones relevantes afines a la pregunta de investigación) y evaluación de la calidad metodológica.

\section{Tipo de estudio}

Metaanálisis de pruebas diagnósticas.

\section{Protocolo de búsqueda y selección de estudio}

Se hizo una búsqueda por especificidad, aplicando la guía Preferred Reporting items for Systematic Reviews and Meta-Analyses (PRISMA) [14] y las recomendaciones de la Colaboración Cochrane [15].

Para la identificación de los estudios, se efectuó una búsqueda de términos en artículos originales publicados en PubMed ${ }^{\circledR}$, SciELO, ScienceDirect ${ }^{\circledR}$ y Google Scholar (al restringir la búsqueda al título, no se obtuvieron resultados), usando la técnica de especificidad (lenguaje controlado, en términos de tesauros: Descriptores en Ciencias de la Salud -DeCS - y Medical Subject Headings - MeSH-). La búsqueda incluyó ocho términos 
para los parámetros de evaluación diagnóstica (False Positives, False Negatives, Negative Predictive Value, Positive Predictive Value, True Negative, True Positive, Sensitivity y Specificity), dos términos para banco de sangre (Blood Banks, Blood Banking) y dos más para donante de sangre (Blood Donation, Blood Donor), obteniendo un total de 32 estrategias de búsqueda diferentes aplicadas en cada base de datos.

En la tamización se incluyeron todos estudios realizados sobre HTLV I/II a partir del año 2000, cuya población de estudio fueron donantes de sangre y que incluyeran los parámetros de validez.

Se excluyeron los estudios de estandarización de pruebas o que no llevaran a cabo evaluaciones diagnósticas con datos completos de sensibilidad y especificidad, o de valores predictivos positivos y negativos (que permitiera identificar el número de resultados falsos y verdaderos, tanto positivos como negativos).

Además, se incluyeron todos los artículos que pasaron la fase anterior. Estos fueron leídos y evaluados para la extracción de las variables de interés, es decir, título, autor, año de publicación, lugar de estudio, tamaño de la población o muestra, tipo de prueba de tamización analizada, verdaderos positivos (donantes HTLV I/II, con resultado positivo en la tamización) y verdaderos negativos (donantes sin HTLV I/II, con resultado negativo en la prueba de tamización), falsos positivos (donantes sin la infección, con resultado positivo en la prueba de tamización) y falsos negativos (donantes con la infección, con resultado negativo en la prueba de tamización).

\section{Reproducibilidad y evaluación de la calidad}

La búsqueda y extracción de las variables fue llevada a cabo por dos revisores; a priori se determinó que las diferencias se resolverían por consenso.

Se aplicó la guía Quality Assessment of Diagnostic Accuracy Studies (QUADAS) 2, para garantizar la calidad metodológica de los estudios incluidos [16].

\section{Plan de análisis}

Se realizó un metaanálisis de efectos aleatorios, mediante el programa Meta-Analysis of Studies of Evaluations of Diagnostic and Screening Tests (MetaDiS) [17].

Se estimó la medida combinada para la sensibilidad, la especificidad, los cocientes de probabilidad positivo y negativo (CPP y CPN), la razón de momios diagnóstica (odds ratio, ORD) y la curva característica operativa del receptor (receiver operating characteristic, ROC) con sus intervalos de confianza (IC) del $95 \%$.

El análisis de heterogeneidad se fundamentó en el estadístico $\mathrm{Q}\left(\chi^{2}\right)$ de Der Simonian-Laird y el $\mathrm{I}^{2}$, y la incertidumbre (sensibilidad) se determinó mediante el porcentaje de peso de cada estudio sobre el resultado global.
Los parámetros se establecieron de manera conjunta para HTLV I/II y luego se hizo metarregresión por tipo de virus (HTLV I y HTLV II).

\section{Resultados}

Se identificaron 427660 artículos, de los cuales se tamizaron 4604. Solo tres artículos cumplieron el protocolo de búsqueda y selección (Figura 1).

Solo uno de los tres estudios utilizó como prueba índice la detección inmunológica; los otros dos usaron PCR (véase Tabla 1). Los estudios fueron realizados en Alemania, Argentina, Austria, España, Francia, Japón y Portugal, en 29099 individuos sanos y 2846 infectados. Los tres estudios analizados presentaron una excelente calidad metodológica, al cumplir con más del $90 \%$ de los criterios de calidad de la guía QUADAS-2.

Las pruebas evaluadas fueron ELISA y quimioluminiscencia, mientras que las pruebas de referencia fueron la PCR y el Western blot.

ELISA permite la detección de antígenos o anticuerpos, mediante el uso de sustrato cromogénicos, cuyas principales ventajas incluyen su rapidez, facilidades de automatización y estandarización; sin embargo, en algunos casos pueden presentar baja sensibilidad analítica.

La quimioluminiscencia parte del mismo fundamento de la ELISA, pero el sustrato es luminiscente y con mayor sensibilidad analítica (1 pg / mL).

En la mayoría de bancos de sangre se emplea ELISA como prueba de tamización de HTLV-1/2. En caso de resultados positivos o indeterminados, se realiza una segunda ELISA, que define si la unidad de sangre debe o no descartarse.

Generalmente, se emplea Western blot para confirmar el estado serológico del donante, cuya positividad varía entre el criterio de la oms y de la Red Europea de Investigación. La primera considera un Western blot positivo cuando se presentan bandas específicas de las proteínas de los genes env, gp46 o gp62/68, o gag, p19, p24 o p53, mientras que la segunda es más exigente, al considerar positivas las muestras con las bandas de p19 y p24 del gen gag, y las de las proteínas de la envoltura viral rgp21 y rgp46-I15.

En general, muchas muestras positivas por ELISA no muestran un patrón completo de bandas para considerarse positivo con Western blot, lo que genera una alta proporción de resultados indeterminados, en cuyo caso se ha recomendado la PCR, que detecta ADN proviral como prueba confirmatoria o complemento a los Western blot indeterminados.

Dos estudios [2,3] evaluaron diferentes ELISA frente a la PCR; en ellos se analizaron 12 subgrupos, con un total de 548 infectados con el HTLV I/II y 5951 individuos 


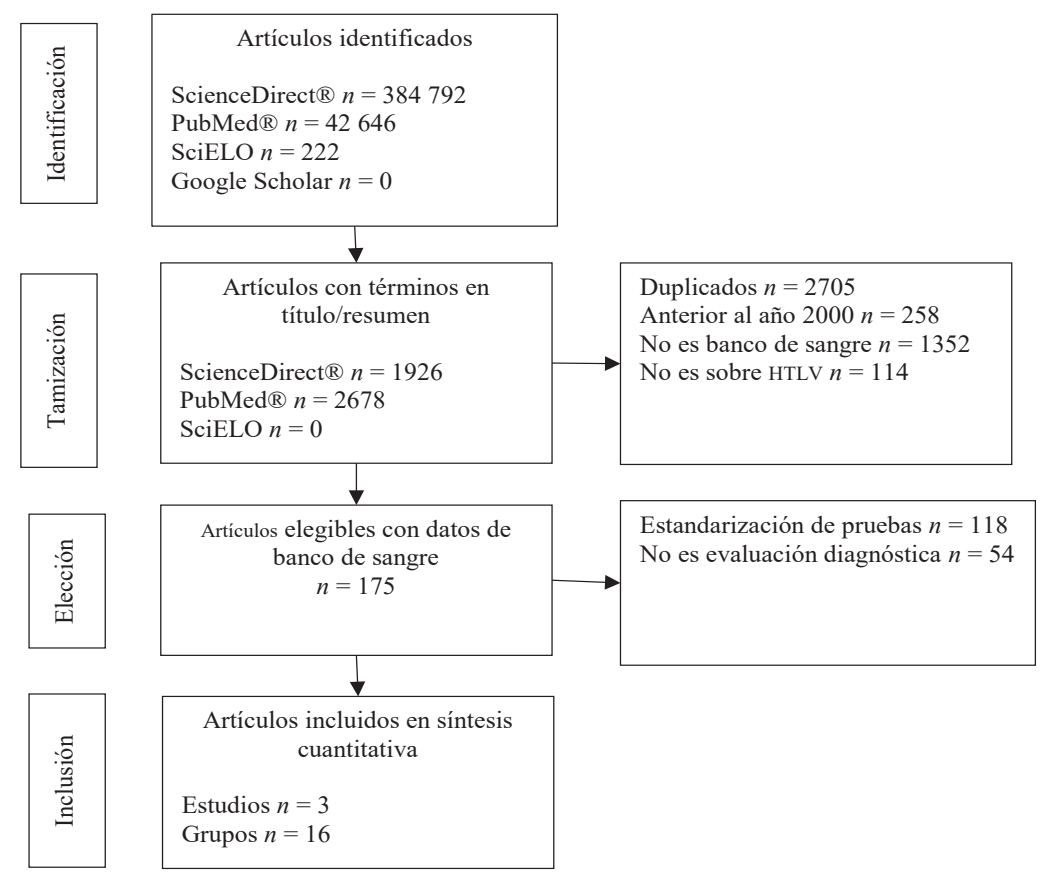

Figura 1. Flujograma de búsqueda y selección de estudios

Tabla 1. Descripción de los estudios que evaluaron la validez de la detección de anticuerpos anti-HTLV

\begin{tabular}{|c|c|c|c|c|c|c|}
\hline Estudio & Año & País & Grupo & $n$ & $\begin{array}{l}\text { Prueba de } \\
\text { referencia }\end{array}$ & Pruebas evaluadas \\
\hline \multirow[t]{3}{*}{ Berini [2] } & \multirow[t]{3}{*}{2008} & \multirow[t]{3}{*}{ Argentina } & Donantes sanos & 305 & \multirow[t]{3}{*}{ PCR Anidada } & \multirow{3}{*}{$\begin{array}{l}\text { Fujirebio, BioKit, } \\
\text { Vironostika y Murex }\end{array}$} \\
\hline & & & Con HTLV I & 86 & & \\
\hline & & & Con HTLV ॥ & 56 & & \\
\hline \multirow[t]{3}{*}{ Kapprell [3] } & \multirow[t]{3}{*}{2010} & \multirow[t]{3}{*}{ Alemania } & Donantes sanos & 5646 & \multirow{3}{*}{$\begin{array}{l}\text { PRISM HTLV I/II, } \\
\text { INNO-LIA, Western } \\
\text { blot, PCR }\end{array}$} & \multirow[t]{3}{*}{ ARCHITECT $r$ HTLV, MureX HTLV } \\
\hline & & & Con HTLV I & 301 & & \\
\hline & & & Con HTLV ॥ & 105 & & \\
\hline \multirow[t]{2}{*}{ Laperche [7] } & \multirow[t]{2}{*}{2017} & \multirow{2}{*}{$\begin{array}{l}\text { Alemania, Austria, España, } \\
\text { Francia, Portugal y Japón }\end{array}$} & Sanos & 23148 & \multirow{2}{*}{$\begin{array}{l}\text { Fujirebio INNO- } \\
\text { LIA® HTLV-I/II }\end{array}$} & \multirow{2}{*}{$\begin{array}{l}\text { Elecsys, Abbott Architect, } \\
\text { Abbott Prism, Ortho Avioq }\end{array}$} \\
\hline & & & Con HTLV I/II & 2298 & & \\
\hline
\end{tabular}

sanos, con los cuales se realizó el metaanálisis para los parámetros de pruebas diagnósticas.

En los 12 subgrupos evaluados, la sensibilidad global fue de $99 \%$ (IC95 \% = 98,0-99,0), la especificidad global fue $100 \%$ (IC95 \% = 99,9-100,0), y sin ningún estudio o subgrupo con mayor peso sobre la medida global, lo que demuestra la pertinencia de la medida combinada, dado que ningún subgrupo sesgó la medida de resumen de estos parámetros (véase Figura 2).

El CPP global fue de 315,8 (IC95 \% = 128,2-778,1) y el CPN global fue de 0,02 (IC95\%=0,01-0,04), sin ningún estudio con un mayor peso sobre la medida combinada (véase Figura 3).
La ORD global fue de 24373 y un área bajo la curva ROC del 99,93\%, con un análisis de sensibilidad que evidenció la robustez de la medida combinada (véase Figura 4).

Al analizar los parámetros para cada tipo de HTLV, se encontró que la sensibilidad global para el HTLV-I fue $100 \%$ (IC95\%=99,0-100,0), y para el HTLV-II, $98 \%($ IC95 \% $=96,0-99,0)$. La especificidad, tanto para el HTLV-I como para el HTLV-II, fue $100 \%$ (IC95\%= 99,0-100,0). El CPP en HTLV-I fue 436,9 (IC95 \% = 91,62083,1), y para el HTLV-II, 210,4 (IC95 \% = 82,1-539,3). El CPN para el HTLV-I fue 0,01 (IC95 \% $=0,00-0,03$ ), y en HTLV-II, 0,03 (IC95\%=0,01-0,07). 

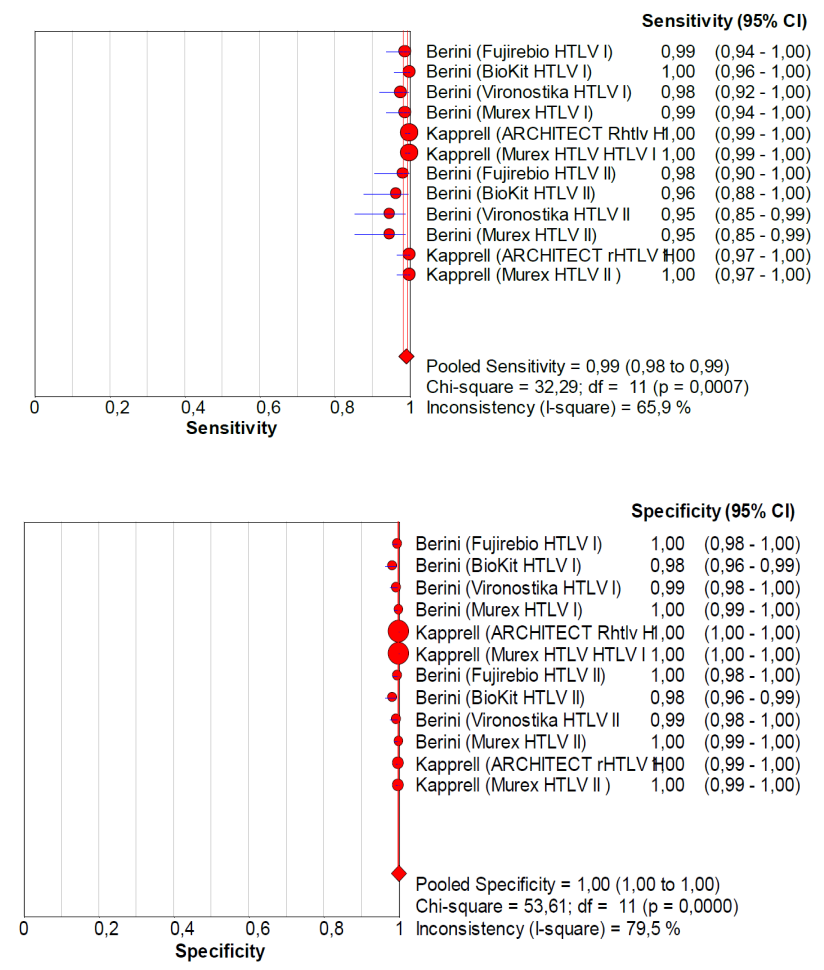

Figura 2. Sensibilidad y especificidad de la tamización serológica
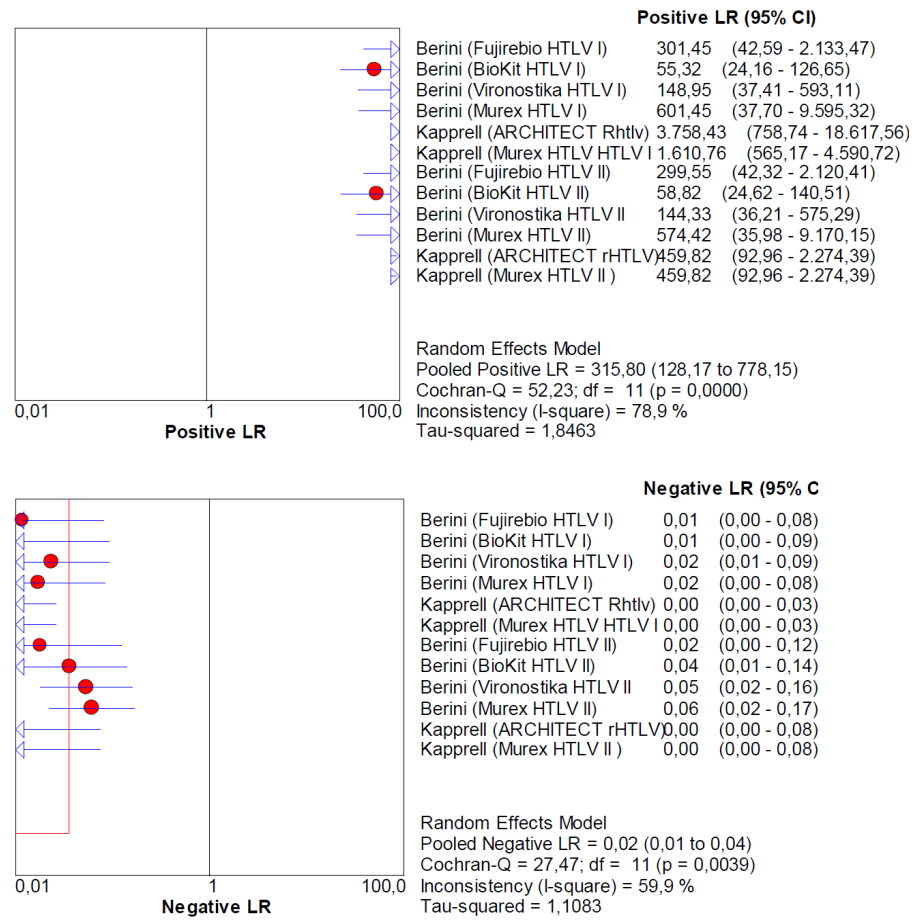

Negative LR $(95 \%$ C

Berini (Fujirebio HTLV I) $\quad 0,01 \quad(0,00-0,08)$ Berini (BioKit HTLVI) $\quad 0,01 \quad(0,00-0,09)$ $\begin{array}{lll}\text { Berini (Vironostika HTLV I) } & 0,02 & (0,01-0,09)\end{array}$ $\begin{array}{ll}\text { Berini (Murex HITVI) } & 0,02 \\ \text { Kapprell (ARCHITECT Rhtlv) } 0,00 & (0,00-0,03)\end{array}$ Kapprell (MUrex HTLV HTLVI0,00 (0,00-0,03) $\begin{array}{rrr}\text { Berini (Fujirebio HTLV II) } & 0,02 & (0,00-0,12)\end{array}$ Berini (BioKit HTLV II) $\quad 0,04 \quad(0,01-0,14)$ $\begin{array}{lll}\text { Berini (Vironostika HTLV II } & 0,05 & (0,02-0,16)\end{array}$ Berini (Murex HTLV II) $\quad 0,06 \quad(0,02-0,17)$ Kapprell (ARCHITECT rHTLV), 00 $(0,00-0,08)$ $\begin{array}{lr}\text { Kapprell (Murex HTLV II ) } & 0,00 \\ (0,00-0,08)\end{array}$

Random Effects Mode Pooled Negative LR $=0,02(0,01$ to 0,04$)$ Cochran- $Q=27,47 ; \mathrm{df}=11(\mathrm{p}=0,0039)$

Inconsistency (I-square) $=59,9 \%$

Tau-squared $=1,1083$

Figura 3. Cocientes de probabilidad de ELISA para HTLV 


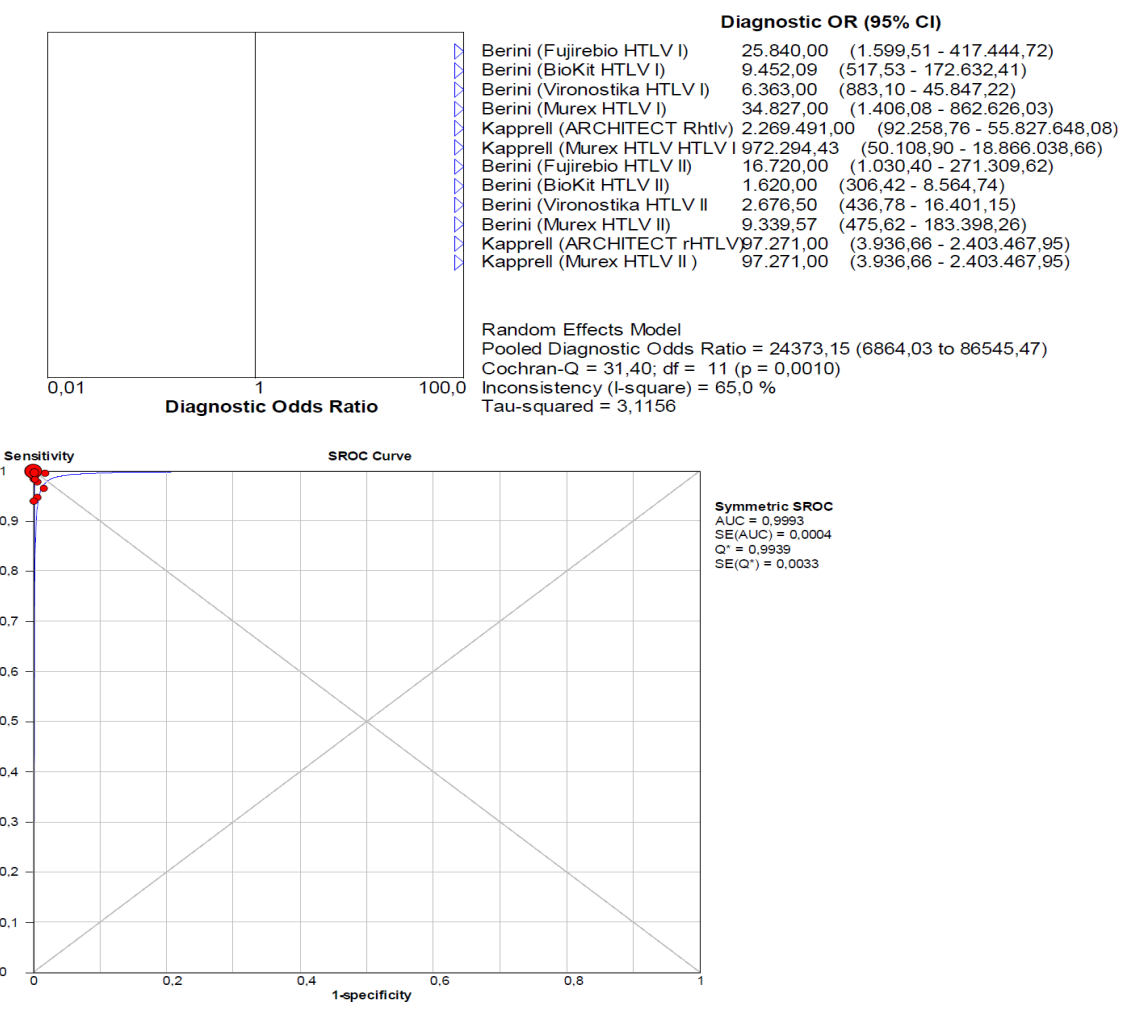

Figura 4. Razón de momios diagnóstica y el área bajo la curva de ELISA para HTLV

La ORD del HTLV-I fue 61200 (IC95 \% = 8550-438 048), y en HTLV-II, 9055 (IC95 \% = 2240-36 612); y el área bajo la curva ROC, tanto para el HTLV-I como HTLV-II, fue $99,9 \%$.

En conjunto, la comparación de los IC demuestra la ausencia de diferencias estadísticamente significativas en los parámetros de evaluación diagnóstica según el tipo de HTLV.

\section{Discusión}

El presente metaanálisis contó con 12 subgrupos, en los cuales se evaluaron pruebas diagnósticas para HTLV I/II frente a la PCR, todos con excelente calidad metodológica en 6643 individuos sanos y 548 infectados, lo que materializa algunas ventajas de este tipo de estudios, como calcular medidas globales sobre los parámetros de evaluación diagnóstica con mejor precisión, actualizar la evidencia disponible en este campo y demostrar el bajo número de estudios sobre evaluación diagnóstica para esta infección en los bancos de sangre. Esto último, posiblemente debido al subdiagnóstico, a la baja prevalencia en algunas regiones y al hecho que la obli- gatoriedad de su tamización en los bancos de sangre es relativamente nueva en muchos países [18].

A lo anterior se suma el hecho de que los pocos estudios sobre evaluación diagnóstica de pruebas para HTLVI/II [2,3,7] se realizaron en Argentina, Japón y algunos países de Europa occidental, de los cuales solo los dos primeros se consideran endémicos; en tal sentido, varios autores han indicado que las regiones endémicas para estos virus incluyen Japón, África subsahariana, América del Sur, el Caribe y algunos focos en Oriente Medio, aunque se desconocen las cifras reales, debido a factores como bajo desarrollo investigativo, problemas de validez de las pruebas diagnósticas (particularmente el registro de altas proporciones de falsos negativos), así como la restricción de su estudio a donantes de sangre, embarazadas y personas hospitalizadas [19,20].

La medida combinada de los grupos analizados demuestra que las pruebas evaluadas tienen una excelente validez diagnóstica, con sensibilidad del $99 \%$ y especificidad del $100 \%$, lo que confirma que la probabilidad de falsos positivos y negativos tiende a cero. El CPP de 315,8 y el CPN de 0,02 demuestran que la ganancia de sensibilidad y de especificidad no se da a expensas de los resultados falsos positivos y falsos negativos respec- 
tivamente. La ORD de 24373 y el área bajo la curva de 99,9\% confirman la excelente capacidad de discriminación entre verdaderos sanos e infectados, así como la excelente relación entre sensibilidad y especificidad, lo que confirma el alto rendimiento de las pruebas de tamización de HTLV I/II [21,22].

Estos resultados son relevantes para las políticas de los bancos de sangre, que en muchos países exigen que toda prueba tamiz reactiva para HTLV I/II debe confirmarse con Western blot, a diferencia de otras infecciones virales (como es el caso del virus de la inmunodeficiencia humana), en las cuales la confirmación de la reactividad se realiza con otro inmunoensayo, diferente al empleado como prueba de tamización inicial [23,24].

Debido a este tipo de evidencia, autores como Moreno et al. [11] consideran importante la implementación de algoritmos alternativos de tamización para HTLV, que incorporen técnicas alternativas, como la aglutinación de partículas de gelatina o quimioluminiscencia como una segunda prueba tamiz, y la técnicas de inmunofluorescencia indirecta como prueba confirmación, debido al alto número de resultados indeterminados arrojados por el Western blot. Sin embargo, para diferentes autores, dichos algoritmos deben modificarse e incorporar pruebas como la PCR desde la tamización, con el fin de reducir el número de muestras reactivas que requieran ser confirmadas y que llevan a un sobrecosto o al diferimiento permanente de los donantes [2,3,11,18,25]. En coherencia con estas controversias, los resultados de este metaanálisis suministran evidencia importante en favor de la aplicación de las pruebas analizadas, aunque el bajo número de estudios restan validez externa y sugieren la necesidad de evaluaciones diagnósticas en otros contextos.

En adición a lo anterior, investigaciones recientes se han ocupado de la morbilidad por HTLV mediante el uso de diferentes pruebas; así, en la actualización de Iwanaga se incluyen estudios que emplearon aglutinación de partículas e inmunofluorescencia [26]; en el análisis que hacen de Cardona et al. se estimó la ocurrencia de la infección mediante quimioluminiscencia y confirmación con Western blot (bandas rgp 46-I, GD21, p19, p24, p26, p28, p32, p36, p53) [27]; el grupo de Yousefi utilizó ELISA [28] y Zhao et al. se sirvieron de quimioluminiscencia y ELISA [29], demostrando la alta diversidad de metodologías para la detección del virus.

Entre las principales limitantes de este estudio se encuentra el bajo número de investigaciones y la ausencia de datos demográficos, epidemiológicos y clínicos que impidieron evaluar la fuente de heterogeneidad en los parámetros evaluados y la realización de metarregresiones $[15,30]$, a lo que se suma el hecho de que los estudios analizados en esta revisión superan los diez años (excepto
Laperche [7]), y sus resultados están supeditados a potenciales cambios en las tecnologías diagnósticas recientes. A pesar de ello, este estudio es relevante para la medicina transfusional, al evidenciar la elevada validez diagnóstica de la ELISA para HTLV I/II, y para la salud pública en general, dado que los bancos de sangre en algunos países constituyen las únicas instituciones que permiten conocer el perfil epidemiológico de esta infección; detectar portadores asintomáticos, en contextos clínicos con gran desconocimiento sobre estos virus por parte del personal médico; brindar tratamiento oportuno a la población afectada; implementar prácticas seguras para minimizar su transmisión y garantizar la inocuidad de las unidades captadas por los bancos de sangre $[6,24]$.

\section{Conclusión}

A pesar del bajo número de estudios incluidos, el excelente tamaño de la muestra de este estudio permite concluir que las pruebas evaluadas cuentan con la validez necesaria para su empleo como prueba tamiz en los bancos de sangre, también permiten garantizar la inocuidad de las unidades captadas durante los procesos de donación y, a su vez, brindan la posibilidad de captar portadores asintomáticos, con el fin de dar manejo oportuno a dicha infección y evitar su transmisión.

\section{Agradecimientos}

A la Escuela de Microbiología de la Universidad de Antioquia.

\section{Fuente de Financiación}

La investigación no tuvo ninguna financiación.

\section{Conflicto de intereses}

Los autores declaran que no existen conflicto de intereses.

\section{Declaración de responsabilidad}

Se declara que los puntos de vista expresados son responsabilidad de los autores y no de la institución en la que trabajan.

\section{Contribución de los autores}

Todos los autores contribuyeron con la idea del estudio, el análisis de datos y la redacción del manuscrito. 


\section{Referencias}

1. Segurado AA, Biasutti C, Zeigler R, et al. Identification of human T-lymphotropic virus type I (HTLV-I) subtypes using restricted fragment length polymorphism in a cohort of asymptomatic carriers and patients with HTLV-I-associated myelopathy/tropical spastic paraparesis from São Paulo, Brazil. Mem. Inst. Oswaldo Cruz. 2002;97(3):329-33. DOI: https://doi.org/10.1590/S007402762002000300009

2. Berini CA, Susana Pascuccio M, Bautista CT, et al. Comparison of four commercial screening assays for the diagnosis of human T-cell lymphotropic virus types 1 and 2. J Virol Methods. 2008;147(2):322-7. DoI: https://doi.org/10.1016/j.jviromet.2007.09.012

3. Kapprell H-P, Stieler M, Oer M, et al. Evaluation of a new thirdgeneration ARCHITECT $r$ HTLV-I/II assay for blood screening and diagnosis. Diagn Microbiol Infect Dis. 2010;67(1):61-69. DoI: https://doi.org/10.1016/j.diagmicrobio.2009.12.021

4. Rivera C, López D, Zamora T, et al. Infección por el virus linfotrópico humano de células T tipo 1 (HTLV-1) y paraparesia espástica. Avances y diagnóstico 35 años después de su descubrimiento. IATREIA. 2017;30(2):146-59. https://doi.org/10.17533/udea.iatreia. v30n2a04

5. Yun S, Kim S, et al. Evaluation of Elecsys HTLV-I/II assay in comparison with ARCHITECT $r$ HTLV-I/II assay with Korean samples. J Clin Lab Anal. 2019; 33(6):e22909. DoI: https://doi.org/10.1002/ jcla.22909

6. Bermúdez-Forero MI, Berrío-Pérez M, Herrera-Hernández AM, et al. Prevalencia de la infección con el virus linfotrópico de células T humanas de tipo 1 y 2 en donantes de sangre en Colombia, 2001-2014: implicaciones sobre la seguridad de la transfusión. Biomédica. 2016;36(Supl. 2):194-200. https://doi.org/10.7705/ biomedica.v36i0.2943

7. Laperche S, Sauleda S, Piron M, et al. Evaluation of sensitivity and specificity performance of Elecsys HTLV-I/II assay in a multicenter study in Europe and Japan. J Clin Microbiol. 2017;55(7):2180-7. DoI: https://doi.org/10.1128/JCM.00169-17

8. Da Silva B, Santos F, Gonçalves N, et al. Performance of commercially available serological screening tests for human T-cell lymphotropic virus infection in Brazil. J Clin Microbiol. 2018;56(12):e00961-18. DoI: https://doi.org/10.1128/ JCM.00961-18

9. Proietti F, Carneiro A, et al. Global epidemiology of HTLV-I infection and associated diseases. Oncogene. 2005;24(39):6058-68. DOI: https://doi.org/10.1038/sj.onc. 1208968

10. Carneiro A, Catalan B, Castro C, et al. HTLv in the Americas: Challenges and perspectives. Rev Panam Salud Pública. 2006;19(1):44-53. DOI: https://doi.org/10.1590/s102049892006000100007

11. Moreno C, Balangero M, Barbás M, et al. Diagnóstico serológico de HTLV-1/2: combinación de técnicas de tamizaje para definir el estatus serológico en donantes de sangre. Rev Argent Microbiol. 2013;45(3):165-8. DoI: https://doi.org/10.1016/S03257541(13)70019-1

12. Morimoto H, Morimoto A, Reiche E, et al. Difficulties in the diagnosis of HTLV-2 infection in HIV/AIDS patients from Brazil: Comparative performances of serologic and molecular assays, and detection of HTLV-2b subtype. Rev Inst Med Trop São Paulo. 2007;49(4):225-30. DoI: https://doi.org/10.1590/s003646652007000400006

13. Cardona J, Higuita L, Ríos Osorio L. Ejecución de revisiones sistemáticas y metaanálisis. En Revisiones sistemáticas de la li- teratura científica: la investigación teórica como principio para el desarrollo de la ciencia básica y aplicada (pp. 27-40). Bogotá: Ediciones Universidad Cooperativa de Colombia; 2016. Dor: http://dx.doi.org/10.16925/9789587600377

14. Moher D, Liberati A, et al. Preferred reporting items for systematic reviews and meta-analyses: The PRISMA Statement. PLos Med. 2009;6(7):e1000097. Dor: https://doi.org/10.1371/journal. pmed.1000097

15. Higgins JPT, Green S (editors). Cochrane Handbook for Systematic Reviews of Interventions Version 5.1.0. London: The Cochrane Collaboration; 2011.

16. Whiting PF, Rutjes AWS, Westwood ME, et al. QUADAS-2: A revised tool for the Quality Assessment of Diagnostic Accuracy Studies. Ann Intern Med. 2011;155(8):529-36. Dor: http://dx.doi. org/10.7326/0003-4819-155-8-201110180-00009

17. Zamora J, Abraira V, Muriel A, et al. Meta-DiSc: A software for meta-analysis of test accuracy data. BMC Med Res Methodol. 2006;6(31) DOI: https://doi.org/10.1186/1471-2288-6-31

18. Biglione M, Berini C. Aportes y consideraciones sobre la infección por los virus linfotrópicos-T humanos tipo 1 y 2 en Argentina. ASEI [internet]. 2013 [citado 2020 jul. 19]; 21(81):84-94. Disponible en: https://www.huesped.org.ar/wp-content/uploads/2014/11/ ASEI-81-84-94.pdf

19. Gessain A, Cassar O. Epidemiological aspects and world distribution of HTLV-1 infection. Front Microbiol. 2012;3:388. DoI: https:// doi.org/10.3389/fmicb.2012.00388

20. Eusebio-Ponce E, Anguita E, et al. HTLV-1 infection: An emerging risk. Pathogenesis, epidemiology, diagnosis and associated diseases. Rev Esp Quimioter. 2019;32(6):485-96. Epub 2019 Oct 25. PMID: 31648512; PMCID: PMC6913074.

21. Torres D, Sierra F, Beltrán OA. Cuando la evidencia evalúa pruebas diagnósticas... ¿Qué debemos saber, qué debemos hacer? Rev Colomb Gastroenterol [internet]. 2004 [citado 2020 jul. 15]; 19(4);281-5. http://www.scielo.org.co/pdf/rcg/v19n4/v19n4a10.pdf

22. Pasamontes J, Cabrera M, Delgado R, et al. Revisión sistemática y metaanálisis de la eficacia diagnóstica de la PET 18F-FDG en recurrencia tumoral de cánceres de cabeza y cuello. Acta Otorrinolaringol Esp. 2008;59(4):190-7. Dor: https://doi.org/10.1016/ S0001-6519(08)73292-8

23. Colombia, Ministerio de Salud y Protección Social. Resolución 437, por la cual se establece la práctica obligatoria de pruebas de anticuerpos contra el virus linfotrópico de células T humanas I/II (HTLV I/II) y la detección de anticuerpos contra el antígeno core del virus de la hepatitis B (Anti HBc) (2014 feb. 14).

24. Herrera A, García M, Bermúdez M. Anexo técnico 4. Circular 082 de 2011. Actualización de algoritmos para pruebas confirmatorias y complementarias (2019-06-14). Instituto Nacional de Salud de Colombia [internet]; 2011 [citado 2020 jun. 25]. Disponible en: https://www.ins.gov.co/Direcciones/RedesSaludPublica/DonacionSangre/NormatividadRelNalBancosSangro/Anexo\%204\%20 Circular\%20082\%20de\%202011.pdf

25. Andrade R, Ribeiro M, Namen M, et al. Evaluation of the use of real-time PCR for human T cell lymphotropic virus 1 and 2 as a confirmatory test in screening for blood donors. Rev Soc Bras Med Trop. 2010;43(2):111-5. DoI: https://doi.org/10.1590/s003786822010000200001

26. Iwanaga M. Epidemiology of HTLV-1 Infection and ATL in Japan: An update. Front Microbiol. 2020;29;11:1124. Dor: https://doi. org/10.3389/fmicb.2020.01124

27. Cardona-Arias JA, Vélez-Quintero C, Calle-González OV, et al. Seroprevalence of human T-lymphotropic virus HTLV and its associated factors in donors of a blood bank of Medellín-Colombia, 
2014-2018. PLoS One. 2019;14(8):e0221060. Dor: https://doi org/10.1371/journal.pone.0221060

28. Yousefi M, Sharifzadeh GR, Ebrahimzadeh A, et al. Prevalence and associated risk factors of HTLV-1 and co-infections of bloodborne viruses in Birjand, Iran's eastern border. Arch Iran Med. 2020;23(10):672-7. DoI: https://doi.org/10.34172/aim.2020.85
29. Zhao J, Zhao F, Han W, et al. HTLv screening of blood donors using chemiluminescence immunoassay in three major provincial blood centers of China. BMC Infect Dis. 2020;20(1):581. Dor: https://doi.org/10.1186/s12879-020-05282-2

30. Oliveros H. La heterogeneidad en los metaanálisis, ¿es nuestra mejor aliada? Rev Colomb Anestesiol. 2015;43(3):176-8. DOI: https://doi.org/10.1016/j.rca.2015.05.002 